\title{
VARIÁVEIS DE MICROFLOTAÇÃO ANALISADAS ESTATISTICAMENTE
}

\author{
J. L. M. REIS*, F. L. SILVA e C. A. PEREIRA \\ Universidade Federal de Ouro Preto \\ jreis_lumia@hotmail.com*
}

Artigo submetido em março/2016 e aceito em outubro/2016

DOI: $10.15628 /$ holos. 2016.4340

\section{RESUMO}

As variáveis aplicadas na microflotação podem apresentar diferentes graus de influência sobre os resultados obtidos com os experimentos. Para que se aumente a compreensão acerca de sua influência estudos estatísticos podem conduzir a dados mais precisos. Dessa forma, os objetivos deste estudo relacionaram-se a (i) entender a significância que as variáveis $\mathrm{pH}$, vazão de gás e tempo de flotação possuem sobre a microflotação (com experimentos conduzidos em duplicatas); (ii) avaliar se existe uma forte correlação entre estas variáveis e (iii) estudar em que faixas granulométricas, dentro das aplicadas no estudo, as partículas sofrem maior arraste hidrodinâmico (para as condições experimentais). Os parâmetros experimentais, cuja definição contou com dados de revisão bibliográfica de estudos publicados, foram: mineral - quartzo puro; surfactante (coletor) dodecilamina; equipamento - tubo de Hallimond com extensor médio - $10 \mathrm{~cm}$ (volume de $250 \mathrm{~mL}$ ); massa dos ensaios $-3,00( \pm 0,05) \mathrm{g}$; concentração do reagente - $10^{-}$ ${ }^{3} \mathrm{~mol}^{-\mathrm{L}^{-1}}$; tempo de condicionamento - $20 \mathrm{~s}$; volume de condicionamento $-250 \mathrm{~mL}$; faixa granulométrica $-53 \mu \mathrm{m}$ a $212 \mu \mathrm{m}$. As análises de Yates e Yates inverso foram aplicadas ao estudo dos dados obtidos. Como resultados obteve-se que a variável pH e as interações da variável pH com a variável vazão de gás são as mais significantes ( $\geq$ $95,0 \%$ de confiabilidade). Constatou-se também que o arraste hidrodinâmico foi maior para as granulometrias próximas a $74 \mu \mathrm{m}$.

PALAVRAS-CHAVE: pH, vazão de gás, tempo de flotação, arraste hidrodinâmico, análise estatística.

\section{MICROFLOTATION VARIABLES ANALYZED STATISTICALLY}

\begin{abstract}
The variables applied in microflotação may present different degrees of influence on the results obtained with the experiments. In order to increase understanding about his influence statistical studies can lead to more accurate data. In this way, the objectives of this study related to (i) understand the significance of the variables $\mathrm{pH}$, gas flow and time of flotation have on the microflotation (with experiments conducted in duplicates); (ii) assess whether there is a strong correlation between these variables and (iii) study on particle size tracks, within the applied in the study, particles suffer greater hydrodynamic drag (for the experimental conditions). The experimental parameters, whose definition included data from literature review of
\end{abstract}

published studies, were: mineral - pure quartz; surfactant (collector) - dodecilamine; equipment Hallimond tube with medium extender $-10 \mathrm{~cm}(250 \mathrm{~mL}$ volume); mass of essays - $3.00( \pm 0.05) \mathrm{g}$; reagent concentration - $10^{-3}$ mol. $\mathrm{L}^{-1}$; conditioning time $-20 \mathrm{~s}$; volume of conditioning $-250 \mathrm{~mL}$; particle size range - 53 $\mu \mathrm{m}$ to $212 \mu \mathrm{m}$. The analyses of Yates method and reverse Yates method have been applied to the study of the data obtained. As a result it was obtained that the variable $\mathrm{pH}$ and $\mathrm{pH}$ variable interactions with the variable gas flow are the most significant ( $\geq 95.0 \%$ of reliability). It was noted also that the hydrodynamic drag was greater for the grain sizes close to $74 \mu \mathrm{m}$

KEYWORDS: $\mathrm{pH}$, gas flow, flotation time, hydrodynamic drag, statistical analysis. 


\section{INTRODUÇÃO}

Reis (et al., 2015) aplicaram a análise estatística de Plackett-Burman para estudo de sete variáveis de microflotação e identificaram como mais influentes o pH, o tempo de flotação e a vazão de gás. Araújo (et al., 2015) estudaram a adsorção do surfactante dodecilamina em quartzo por meio de microflotação e medidas do potencial zeta (variando pH e concentração do reagente), concluindo que, pelas medidas de potencial zeta, ocorre a reversão de carga medida no plano de cisalhamento. Quanto aos experimentos de microflotação, para pH na faixa ácida e neutra, a flotabilidade do mineral estudado sofreu incrementos com a elevação da concentração do coletor até a concentração micelar crítica do reagente, e decresceu após este ponto. Com relação a faixa básica de pHs, a flotabilidade manteve-se constante. Guimarães Júnior (et al., 2015) analisaram a influência da granulometria no arraste hidrodinâmico, chegando a conclusão que este é maior nas faixas de tamanho intermediárias a $212 \mu \mathrm{m}$ e $180 \mu \mathrm{m}$ (para o mineral estudado - apatita). Segundo os autores isso é devido ao fato destas partículas não serem pequenas o suficiente (pouca massa) para serem levadas pelo fluxo de água descendente, gerado pela ascensão das bolhas de gás, e nem possuir dimensão tão significante (maior massa) a ponto de sua massa permitir que elas permaneçam estáveis no meio aquoso e possam até sofrer colisão com as bolhas. Assim, o seu tamanho intermediário lhe confere um comportamento diferenciado, que faz com que essas partículas sejam aprisionadas pela zona de baixa pressão que se forma atrás das bolhas em ascensão, sendo arrastadas pela mesma.

As probabilidades de colisão, adesão e transporte governam a recuperação das partículas minerais pela flotação verdadeira. Cada uma destas probabilidades também sofre influência do tamanho das partículas, onde as partículas menores, devido a influência das linhas de fluxo hidrodinâmico ao redor das bolhas ascendentes (que acabam por dificultar a colisão destas com a interface gás-líquido na película da bolha) levam a menor probabilidade de colisão. Em se tratando das partículas de maior dimensão as probabilidades de adesão e transporte acabam sendo influenciadas por sua maior inércia, levando a um menor tempo de contato e, dessa forma, redução destas probabilidades. (BALTAR, 2010; DA SILVA, 2015). Porém, nem só a flotação verdadeira influencia na presença de partículas na fração flotada. $O$ arraste hidrodinâmico, o arraste por oclusão em agregados e o arraste por slime coating também são responsáveis pelo direcionamento das partículas a fração flotada. (TABOSA, 2007). Rodrigues (et al., 2011) estudaram as variáveis granulometria, agitação, vazão de gás e uso de extensor para conhecer quais suas influências sobre o arraste hidrodinâmico e concluíram que a granulometria é o parâmetro que mais afeta este fenômeno, em termos quantitativos.

Martins (et al., 2002) estudaram o efeito causado pela da distribuição granulométrica do quartzo na flotação e obtiveram como resultados que faixas granulométricas mais restritas favorecem a flotabilidade e que granulometrias mais amplas dificultam a recuperação das frações mais grosseiras. Ulusoy (et al., 2003) estudaram a forma, estrutura e molhabilidade de partículas de quartzo e concluíram que a molhabilidade (tensão superficial) aumenta com o aumento da rugosidade e esfericidade deste mineral. Guven (et al., 2015) estudaram a influência da rugosidade e forma de partículas de quartzo em sua flotabilidade, através de microflotação, e concluíram que superfícies mais angulosas e rugosas favorecem a flotabilidade deste mineral. 
Para que a flotação verdadeira pudesse ser a utilizada na análise estatística, conduzida pelo algoritmo de Yates e Yates inverso - Yates $(1935 ;$ 1937), o arraste hidrodinâmico foi avaliado para cada condição aplicada aos experimentos. Barcellos (et al., 2009) utilizaram está metodologia na otimização de ensaios de jigagem e puderam conhecer quais as variáveis estudadas interagiam e, também, a natureza dessas interações (possibilitando definir padrões para que o conjunto de variáveis levasse a um resultado otimizado). A mesma metodologia foi empregada com sucesso no estudo de Vicario (1996).

Os objetivos do estudo foram: (i) entender a significância que as variáveis $\mathrm{pH}$, vazão de gás e tempo de flotação possuem sobre a microflotação (com experimentos conduzidos em duplicatas); (ii) avaliar se existe uma forte correlação (interações significativas) entre estas variáveis e (iii) estudar em que faixas granulométricas, dentro das aplicadas no estudo, as partículas sofrem maior arraste hidrodinâmico (para as condições experimentais).

\section{MATERIAIS E MÉTODOS}

A preparação da amostra de quartzo para os experimentos contou com cominuição em moinho vibratório de panela (FRITSCH pulverisette 9), peneiramento à úmido para adequação à faixa granulométrica do estudo (- $212 \mu \mathrm{m}$ e $+53 \mu \mathrm{m})$ e lixiviação ácida $(\mathrm{HCl} 36,5 \%)$ que pretendia eliminar possíveis impurezas superficiais. Para remoção dos íons $\mathrm{H}^{+}$na superfície da amostra, a mesma foi lavada com água destilada $(A D)$ até que as medidas de $\mathrm{pH}$ (pHmetro digital DIGIMED $\mathrm{DM}-22$ ) da água de lavagem apresentassem leituras na faixa de $\mathrm{pH}$ neutra. Tendo sido obtidas as leituras de $\mathrm{pH}$ em faixa neutra a amostra passou por secagem em estufa (TECNAL TE-394/3) a $100^{\circ} \mathrm{C}\left( \pm 5^{\circ} \mathrm{C}\right)$ e resfriamento até a temperatura ambiente. Inicialmente foram realizados estudos da pureza do mineral adotado. Dessa forma desenvolveram-se análises mineralógica (difração de raios $X$ - difratômetro de raios $X$ Rigaku, série $D / M a x-B$; método do pó total) e química semiquantitativa (fluorescência de raios $X$ - espectrômetro de fluorescência de raios $X$ por energia dispersiva EDX-720).

Para a definição dos níveis máximos e mínimos necessários à condução dos experimentos com as variáveis testadas utilizaram-se dados de artigos correlatos. Na vazão de gás aplicou-se nível máximo entre $55( \pm 5) \mathrm{cm}^{3} / \mathrm{min}$ (RODRIGUES et al., 2010) e nível mínimo compreendido entre $35( \pm 5) \mathrm{cm}^{3} / \mathrm{min}$ (PINHEIRO et al., 2010). O pH foi definido pela constante ácida ( $\mathrm{pH} \sim 3,5$ ), no caso do nível mínimo, e constante básica $(\mathrm{pH} \sim 10,5)$, para o nível máximo, do reagente empregado (DDA) (SILVA, 2008). Para a microflotação definiu-se como nível máximo 5 minutos (MANGRAVITE JÚNIOR et al.,1972) e nível mínimo 1 minuto (PINHEIRO et al., 2010).

Alguns parâmetros envolvidos nos estudos de microflotação foram fixados, quais sejam: tempo de condicionamento da amostra de 20 s (FEASBY, 1966); o volume de condicionamento da amostra de $250 \mathrm{~mL}$ (volume do tubo de Hallimond modificado empregado), massa de quartzo para os ensaios de 3,00 ( $\pm 0,05)$ gramas (REIS, et al., 2015); a concentração de dodecilamina de $10^{-3}$ mol. $L^{-1}$, baseada na mínima concentração com considerável recuperação mássica no estudo de Araújo et al. (2015); faixa granulométrica de $53 \mu \mathrm{m}$ a $212 \mu \mathrm{m}$ para conhecimento das dimensões compreendidas entre esses limites que são mais susceptíveis ao arraste hidrodinâmico. Na Tabela 1 apresentam-se dados relativos ao planejamento estatístico experimental. 
Tabela 1. Planejamento estatístico experimental

\begin{tabular}{cccc}
\hline \multirow{2}{*}{ Experimento } & \multicolumn{3}{c}{ Variáveis estudadas } \\
\cline { 2 - 4 } & pH [-] & Vazão de gás [cm $\mathbf{3}$ /min] & Tempo de microflotação [min] \\
\hline$T$ & $3,5( \pm 0,5)$ & $35( \pm 5)$ & 1 \\
$A$ & $10,5( \pm 0,5)$ & $35( \pm 5)$ & 1 \\
$B$ & $3,5( \pm 0,5)$ & $35( \pm 5)$ & 5 \\
$A B$ & $10,5( \pm 0,5)$ & $35( \pm 5)$ & 5 \\
C & $3,5( \pm 0,5)$ & $55( \pm 5)$ & 1 \\
$A C$ & $10,5( \pm 0,5)$ & $55( \pm 5)$ & 5 \\
$B C$ & $3,5( \pm 0,5)$ & $55( \pm 5)$ & 5 \\
$A B C$ & $10,5( \pm 0,5)$ & $55( \pm 5)$ & 5 \\
\hline
\end{tabular}

Fonte: Dados do estudo.

Os estudos de arraste hidrodinâmico foram desenvolvidos nas quatro condições empregadas nos experimentos, porém sem a presença do coletor na água destilada (assegurando que a presença de partículas de quartzo no flotado fosse devida ao arraste hidrodinâmico). Dessa forma o tempo de condicionamento (agitação) foi conduzido por $20 \mathrm{~s}$, o tempo de flotação por 1 minuto e 5 minutos e a vazão de gás regulada em $35( \pm 5) \mathrm{cm}^{3} / \mathrm{min}$ e $55( \pm 5) \mathrm{cm}^{3} / \mathrm{min}$. Após os ensaios a fração flotada e afundada foram filtradas, secas em estufa, resfriadas até a temperatura ambiente e pesadas. A fração flotada foi peneirada com as peneiras da série Tyler e repesada para avaliação do arraste hidrodinâmico por faixa granulométrica ( $212 \mu \mathrm{m} ; 147 \mu \mathrm{m} ; 104 \mu \mathrm{m} ; 74 \mu \mathrm{m} ; 53$ $\mu \mathrm{m})$.

Para o preparo do reagente coletor (cloreto de dodecilamina a $10^{-3} \mathrm{~mol}^{-\mathrm{L}^{-1}}$ ), foram pesadas 0,3783 g de DDA sólida (SIGMA-ALDRICH Brasil Ltda.), adicionadas a um béquer de $500 \mathrm{~mL}$ que foi preenchido com $300 \mathrm{~mL}$ de AD. Para que se obtivesse uma neutralização 1:1, 0,1971 g de $\mathrm{HCl} 36,5$ $\%$, foram adicionadas a este béquer. Utilizou-se agitação e aquecimento ( $\leq 60 \stackrel{\circ}{ }$ ) visando a promover a solubilização do sólido. Adicionou-se essa solução a um balão volumétrico de $2 \mathrm{~L}$ que foi preenchido com $A D$ até a aferição do menisco. A solução foi submetida a agitação por 5 minutos com agitador magnético. Para que o volume de coletor fosse adequado a todos os experimentos planejados esses procedimentos foram refeitos uma vez.

Soluções de $\mathrm{NaOH}$ e $\mathrm{HCl}$ foram aplicadas em diversas diluições $(0,1$ \%; 0,5 \%; 1,0 \%; 5,0 \% e $10,0 \%$ - peso/volume e volume por volume, respectivamente) para que o pH das soluções de DDA fosse adequado às faixas predefinidas para os experimentos. Essas adequações eram conduzidas em béqueres com agitador magnético enquanto as leituras eram efetuadas pelo pHmetro digital, previamente calibrado.

Os procedimentos para os experimentos de microflotação eram: adicionar $3,0( \pm 0,005) \mathrm{g}$ da amostra de quartzo puro ao tubo de Hallimond modificado com extensor; colocar a solução de DDA, na concentração e pH apropriados e iniciar a agitação magnética por $20 \mathrm{~s}$ (a velocidade foi padronizada naquela que permitia que as partículas ficassem suspensas). Na sequência era introduzido o $\mathrm{N}_{2}(\mathrm{~g})$, com a vazão predefinida, durante o templo planejado. Terminada a microflotação, os produtos flotado e afundado, foram recolhidos em béqueres, filtrados à vácuo (Papel filtro Qualy com $14 \mu \mathrm{m}$ de poros; Bomba à vácuo TECNAL TE-0581), passaram por secagem em estufa, resfriamento até a temperatura ambiente e pesagem (Balança SHIMADZU AUW220D) para obtenção dos dados de recuperação mássica.

A análise de Yates foi aplicada na análise dos resultados. As equações são apresentadas a seguir: 


$$
D M=\frac{Y-n}{2^{n}}
$$

Onde: $\mathrm{DM}$ - diferença média [-]; $\mathrm{n}-\mathrm{n}^{\circ}$ de níveis [-].

$$
\phi_{u}=\text { níveis }-1
$$

Onde: $\varphi_{u}-\mathrm{n}$ o de graus de liberdade unitário [-].

$$
\phi_{\exp }=\text { unid.experimentais } \times \phi_{u}
$$

Onde: $\varphi_{\exp }-\mathrm{n}$ o de graus de liberdade do experimento [-].

$$
\sigma_{\exp }=\sqrt{\frac{\sum\left(T_{1}-T_{2}\right)^{2}}{2 \times \phi_{\exp }}}
$$

Onde: $\sigma_{\exp }$ - desvio padrão do conjunto dos experimentos [-]; $\mathrm{T}_{1}$ - respostas dos experimentos [-]; $\mathrm{T}_{2}-$ respostas das duplicatas [-].

$$
E P M_{i}=E P M_{s}=\frac{\sigma_{\text {exp }}}{\sqrt{N}}
$$

Onde: $\mathrm{EPM}_{\mathrm{i}}$ - erros padrões de média inferior [-]; $\mathrm{EPM}_{\mathrm{s}}$ - erros padrões de média superior []; $\mathrm{N}-\mathrm{n}$ o total de resultados [-].

$$
E P D M=\sqrt{E P M_{i}^{2}+E P M_{s}^{2}}
$$

Onde: EPDM - erro padrão da diferença média [-].

$$
\delta_{c a l}=\frac{D M}{E P D M}
$$

Onde: $\delta_{\text {cal }}-$ significância estatística calculada [-].

$$
\delta_{c a l}>\delta_{t a b}
$$

Onde: $\delta_{\text {tab }}-$ significância estatística tabelada (tabela $\boldsymbol{t}$ de Student) [-]. 


\section{RESULTADOS E DISCUSSÃO}

Na Tabela 2 podem ser observados os resultados obtidos com a análise química semiquantitativa:

Tabela 2. Resultados da análise química semiquantitativa da amostra de quartzo

\begin{tabular}{ccccc}
\hline $\mathrm{SiO}_{2}[\%]$ & $\mathrm{SO}_{3}[\%]$ & $\mathrm{Fe}_{2} \mathrm{O}_{3}[\%]$ & $\mathrm{CuO}[\%]$ & $\mathrm{GeO}_{2}[\%]$ \\
\hline 98,907 & 1,063 & 0,016 & 0,009 & 0,005 \\
\hline
\end{tabular}

Fonte: Dados obtidos com a análise química semi-quantitativa.

Os dados acima evidenciam que o quartzo $\left(\mathrm{SiO}_{2}\right)$ é o principal componente da amostra e que os demais óxidos analisados possuem proporções insignificantes.

Na Figura 1 são apresentados os resultados da difratometria de raios $X$ da amostra empregada nos experimentos:

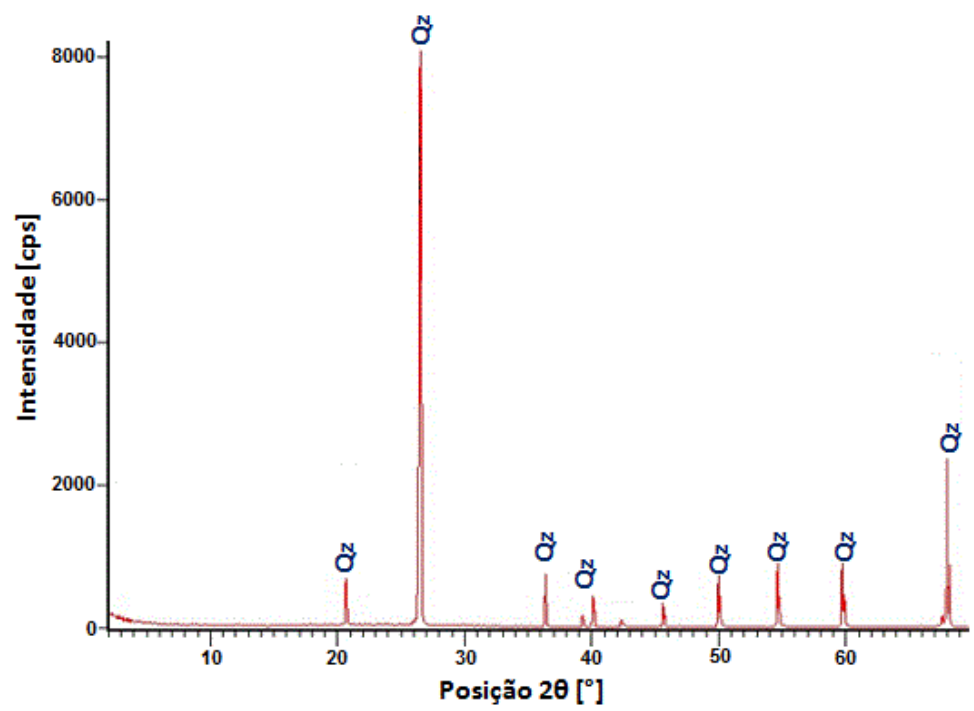

Figura 1. Difratograma de raios $X$ da amostra de quartzo empregada. Observações: $Q z$ - quartzo; ânodo de cobre. Fonte: Dados da análise mineralógica por difração de raios $X$.

Apenas picos característicos do mineral quartzo foram encontrados, confirmando os dados da análise química semi-quantitativa, cuja a interpretação do difratograma se deu pelo software Jade 7.0 (MDI).

Os dados dos experimentos e suas duplicatas, com reprodutibilidade nos experimentos, são apresentados na Figura 2, juntamente aos dados de arraste hidrodinâmico: 


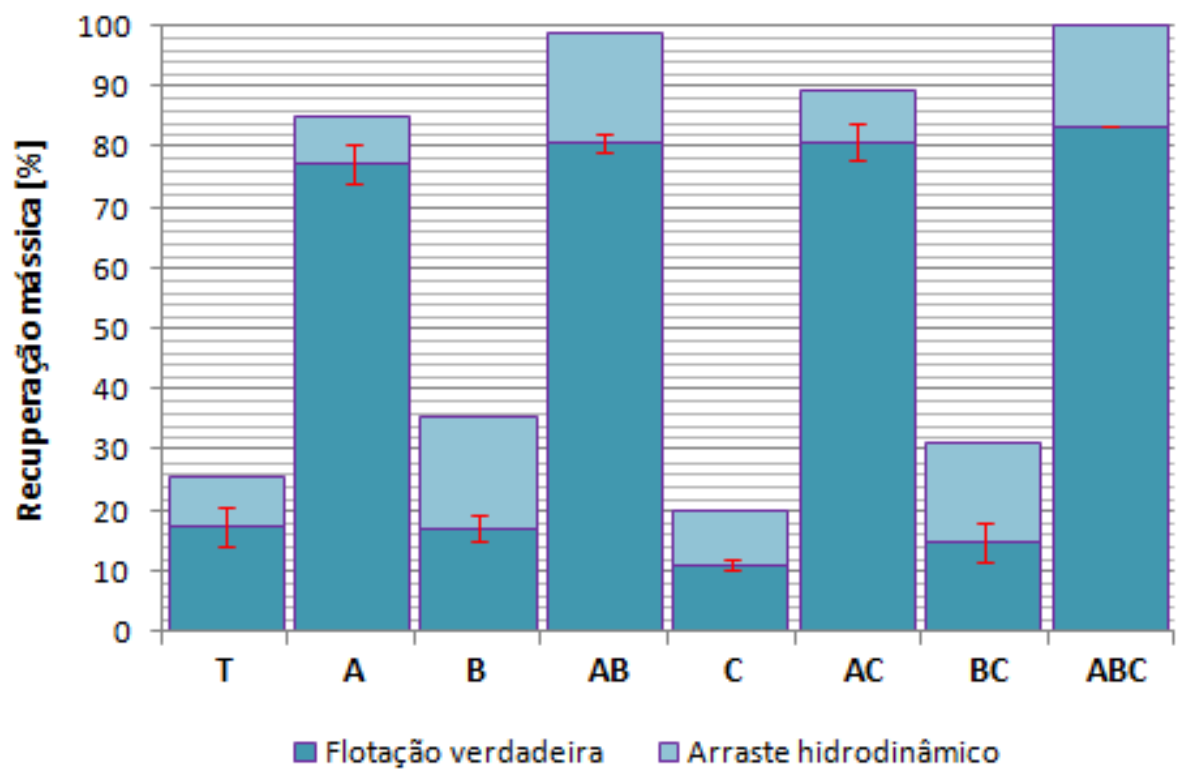

Figura 2. Gráfico da recuperação por flotação verdadeira e por arraste hidrodinâmico. Fonte: Dados experimentais.

De acordo com os dados de recuperação mássica por flotação verdadeira destacam-se os experimentos $A B C, A C$ e $A B$. A média do desvio padrão dos dados de recuperação mássica foi de 2,17 pontos percentuais e de arraste hidrodinâmico 2,47 pontos percentuais.

Os dados de arraste hidrodinâmico analisado por faixa granulométrica são apresentados na Figura 3:

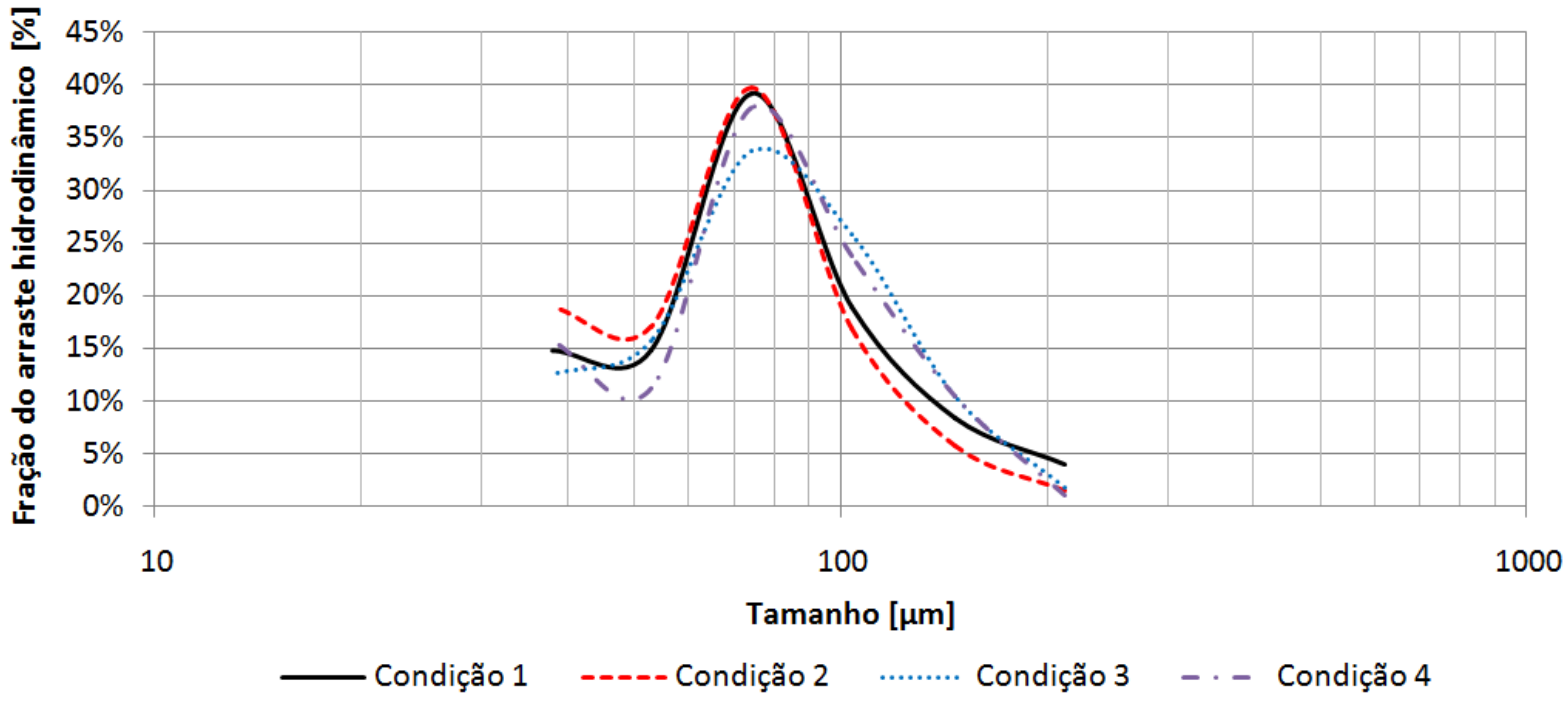

Figura 3. Gráfico da avaliação do arraste hidrodinâmico por faixa granulométrica. Observações: condição 1 (experimentos C e AC); condição 2 (experimentos BC e ABC), condição 3 (experimentos T e A) e condição 4 (experimentos B e AB). Fonte: Dados experimentais.

As partículas do mineral analisado por faixa granulométrica mostraram comportamento semelhante para as quatro condições experimentais do estudo (de vazão de gás e tempo de flotação avaliadas). As partículas maiores apresentaram menor arraste hidrodinâmico. Esse fenômeno foi sofrendo incrementos com a redução do tamanho das partículas até a faixa granulométrica intermediária a $70 \mu \mathrm{m}$ e $80 \mu \mathrm{m}$, abaixo da qual a situação se inverte, maior se torna o arraste com o decréscimo da granulometria. Essa tendência sofre nova reversão próximo a 50 
$\mu \mathrm{m}$ em 75 \% das condições testadas (apenas na condição 3 não se comprovou a mudança de comportamento).

A análise das curvas da Figura 3 e dos dados da Figura 2 permite as seguintes inferências para o mineral em questão: (i) o tempo de flotação e a vazão de gás, nas condições testadas, não levaram a diferenças muito pronunciadas na porcentagem de arraste granulométrico por faixa, apresentando diferenças apenas na massa arrastada; (ii) as partículas maiores que $100 \mu \mathrm{m}$, provavelmente por apresentarem maior massa, não são tão influenciadas por esse fenômeno e podem se manter estáveis no meio aquoso até que sejam atingidas e coletadas por bolhas de gás; (iii) as partículas próximas a $74 \mu \mathrm{m}$ são as mais influenciadas pelo arraste hidrodinâmico possivelmente por serem as mais susceptíveis a aprisionamento pela zona de baixa pressão que ocorre abaixo das bolhas de gás e que permite a ascensão dessas partículas até a região de coleta do flotado mesmo que elas não tenham sido coletadas; (iv) para este mineral as melhores faixas granulométricas para estudos de microflotação são aquelas abaixo de $60 \mu \mathrm{m}$ ou acima de $100 \mu \mathrm{m}$, devido ao fato de serem as menos influenciadas pelo fenômeno em discussão.

A análise de Yates foi empregada para a avaliação de quais as variáveis eram significativas e se existiam interações significativas ( $\geq 95 \%$ ) entre elas. Os resultados foram: $\Sigma\left(T_{1}-T_{2}\right)^{2}=99,47$; $\sigma_{\text {exp }}=2,49 ; \mathrm{EPM}= \pm 0,88 ; \mathrm{EPDM}=1,25 ; \delta t a b=2,306$ (ROJAS, 2009). A análise dos resultados obtidos é apresentada pela Tabela 3:

Tabela 3 - Algoritmo de Yates. Fonte: Dados experimentais.

\begin{tabular}{|c|c|c|c|c|c|c|c|c|c|c|c|}
\hline Experimento & R1 & R2 & $R 1+R 2$ & Y-1 & $\mathrm{Y}-2$ & $\mathrm{Y}-3$ & DM & R1-R2 & $\left(\right.$ R1-R2) ${ }^{2}$ & Scalc & Significância \\
\hline$T$ & 15,06 & 19,86 & 34,92 & 189,35 & 385,09 & 765,78 & 95,72 & $-4,80$ & 23,04 & $T$ & - \\
\hline$A$ & 79,60 & 74,83 & 154,43 & 195,74 & 380,69 & 523,74 & 65,47 & 4,77 & 22,75 & 52,51 & $\mathrm{~S}$ \\
\hline B & 18,62 & 15,59 & 34,21 & 184,07 & 246,83 & 18,94 & 2,37 & 3,03 & 9,18 & 1,90 & $\mathrm{~N}$ \\
\hline$A B$ & 79,73 & 81,80 & 161,53 & 196,62 & 276,91 & 5,74 & 0,72 & $-2,07$ & 4,28 & 0,58 & $\mathrm{~N}$ \\
\hline $\mathrm{C}$ & 10,66 & 11,63 & 22,29 & 119,51 & 6,39 & $-4,40$ & $-0,55$ & $-0,97$ & 0,94 & $-0,44$ & $\mathrm{~N}$ \\
\hline$A C$ & 83,05 & 78,73 & 161,78 & 127,32 & 12,55 & 30,08 & 3,76 & 4,32 & 18,66 & 3,02 & S \\
\hline $\mathrm{BC}$ & 12,53 & 17,07 & 29,60 & 139,49 & 7,81 & 6,16 & 0,77 & $-4,54$ & 20,61 & 0,62 & $\mathrm{~N}$ \\
\hline$A B C$ & 83,51 & 83,51 & 167,02 & 137,42 & $-2,07$ & $-9,88$ & $-1,24$ & 0,00 & 0,00 & $-0,99$ & $\mathrm{~N}$ \\
\hline
\end{tabular}

Pela análise desenvolvida detectaram-se como variáveis significativas, a nível de $95 \%$ ou superior, a variável do experimento $\mathrm{A}(\mathrm{pH}$ - recuperação mássica média: 77,22 \%) e as variáveis do experimento $A C$ ( $\mathrm{pH}$ e vazão de gás - recuperação mássica média: 80,89 \%). Comparando-se com os melhores resultados de recuperação mássica percebe-se que apenas o experimento AC, que apresentou uma recuperação mássica média de $80,89 \%$, apareceu entre os três melhores resultados $(A B C, A C$ e $A B)$. Convém observar também que o experimento $A B C$ que teve a melhor recuperação mássica média $(83,51 \%)$ não obteve a significância estatística adotada e sim 60 \% de confiabilidade. Outra constatação é que apesar de uma variável ser significativa ao nível do estudo isso não se estabelece como única condicionante para que se obtenha a melhor recuperação mássica quando a variável é a única utilizada em nível máximo (como foi o caso do experimento A).

O Yates inverso foi aplicado para o conhecimento do significado das variáveis significativas em interação ( $A C$ - pH e vazão de gás). No Quadro 1 é apresentada esta análise e na Figura 4 o gráfico de análise das interações de variáveis significativas: 
Quadro 1 - Aplicação do Yates inverso. Fonte: Dados experimentais.

\begin{tabular}{|c|c|c|c|c|c|c|c|c|}
\hline \multicolumn{5}{|c|}{ Yates inverso } & \multicolumn{4}{|c|}{ Quadro de variáveis resposta } \\
\hline Efeito & DM & Y-1 & Y-2 & $(Y-2) / 2$ & \multirow{2}{*}{ Variáveis } & \multirow{2}{*}{\multicolumn{3}{|c|}{ (A) $\mathrm{pH}$}} \\
\hline$A C$ & 3,76 & 3,21 & 164,4 & 82,2 & & & & \\
\hline $\mathrm{C}$ & $-0,55$ & 161,19 & $-25,94$ & $-12,97$ & \multirow{3}{*}{ (C) Vazão de gás } & Níveis & $3,5( \pm 0,5)$ & $10,5( \pm 0,5)$ \\
\hline $\mathrm{A}$ & 65,47 & 4,31 & $-157,98$ & $-78,99$ & & $35( \pm 5) \mathrm{cm}^{3} / \mathrm{min}$ & 17,28 & $-78,99$ \\
\hline $\mathrm{T}$ & 95,72 & $-30,25$ & 34,56 & 17,28 & & $55( \pm 5) \mathrm{cm}^{3} / \mathrm{min}$ & $-12,97$ & 82,2 \\
\hline
\end{tabular}

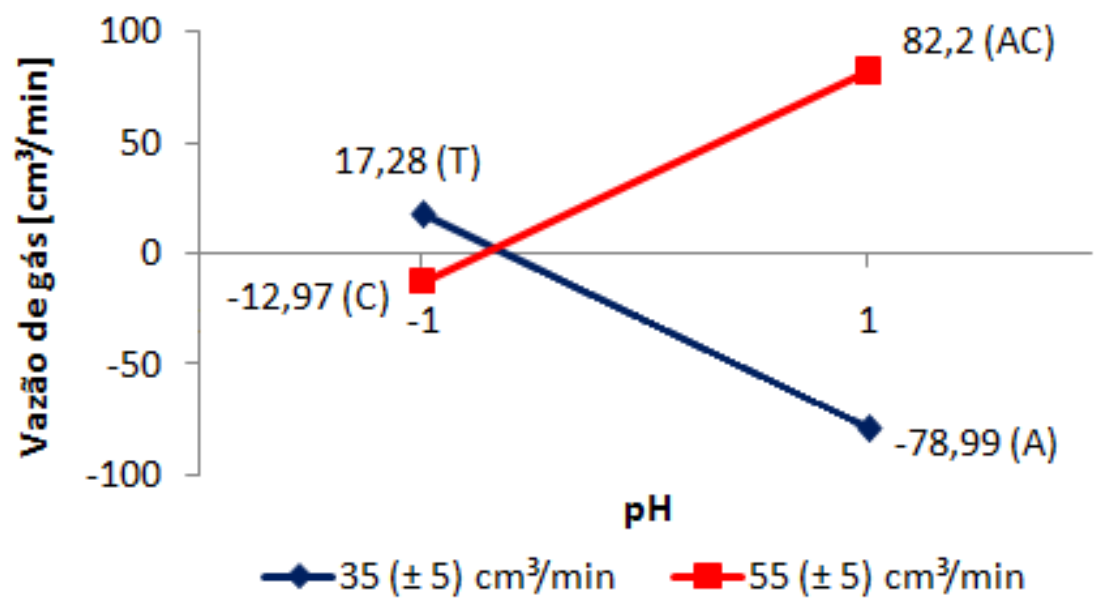

Figura 4. Gráfico de análise das interações de variáveis significativas. Observações: '-1' = variável em nível mínimo; ' 1 ' = variável em nível máximo. Fonte: Dados experimentais.

Através dos dados obtidos com a análise por Yates inverso percebe-se que: com a variável vazão de gás em nível mínimo - o pH, ao passar de '-1' (nível mínimo) para '1' (nível máximo), leva a grande redução na recuperação mássica. Quando a variável vazão de gás se encontra em nível máximo - o pH ao passar de '-1' para ' 1 ', permite considerável aumento de recuperação mássica. Dessa forma está confirmado que a otimização do resultado é obtida com as duas variáveis ( $\mathrm{pH}$ e vazão de gás) interagindo em nível máximo.

\section{CONCLUSÕES}

A significância das variáveis de microflotação $(\mathrm{pH}$, vazão de gás e tempo de flotação) pode ser estudada através do algoritmo de Yates e mostrou que o pH (experimento $A$ ) e as interações entre o pH e a vazão de gás (experimento $\mathrm{AC}$ ) possuem a maior significância (forte correlação), neste caso. Esses dados estão de acordo com os encontrados por Azevedo Filho (et al., 2013). Através da análise de Yates inverso, constata-se que as interações das variáveis $\mathrm{pH}$ e vazão de gás, ambas em nível máximo, fornecem resultados otimizados de recuperação mássica.

O maior arraste hidrodinâmico foi detectado nas faixas granulométricas próximas a $74 \mu \mathrm{m}$.

Convém observar que, ao comparar-se os melhores resultados de recuperação mássica (experimentos $A B C, A C$ e $A B$ ) aos dados das variáveis ou interações de variáveis com $95 \%$ de significância estatística $(A$ e $A C$ ) é notório que apenas as variáveis do experimento $A C$ obtiveram destaque entre as de maior recuperação mássica. Dessa forma podemos constatar que mesmo sendo significativa ao nível do estudo ( $\geq 95,0 \%$ de confiabilidade) isso não se estabelece como única condicionante para que se obtenha a melhor recuperação mássica quando a variável é a 
única utilizada em nível máximo (como foi o caso do experimento $A$, com a quarta melhor recuperação mássica).

\section{REFERÊNCIAS BIBLIOGRÁFICAS}

1. ARAÚJO, A. C. A.; REIS, J. L. M.; RODRIGUES, O. M. S. Estudo da adsorção de dodecilamina na superfície do quartzo. In: ENTMME - Encontro Nacional de Tratamento de Minérios e Metalurgia Extrativa, 26, 2015, Poços de Caldas, MG. ENTMME: Volume II/Org. Daniela Gomes Horta et al. Anais... São Paulo - SP: Associação Brasileira de Tratamento de Minérios e Metalurgia Extrativa, v. 2, p. 501-509, 2015.

2. AZEVEDO FILHO, J.B.; SOUZA, R.F.; MELO, J.V.; PAULO, J.B.A. Avaliação da eficiência na flotação de quartzo e feldspato potássico utilizando planejamento fatorial. In: ENTMME - Encontro Nacional de Tratamento de Minérios e Metalurgia Extrativa, 25, 2013, Goiânia, GO. ENTMME: Volume 1/Org. André Carlos Silva e Elenice Maria Schons Silva. Anais... São Paulo - SP: Associação Brasileira de Tratamento de Minérios e Metalurgia Extrativa, v. 1, p. 53-60, 2013.

3. BALTAR, C. A. M. Flotação no tratamento de minérios. 2. ed. Recife: Editora Universitária da UFPE, 2010. $232 \mathrm{p}$.

4. BARCELOS, H. O.; ROCHA, G. M.; PERES, A. E. C.; PEREIRA, C. A. Análise estatística utilizando o algoritmo Yates para otimização de ensaios de jigagem. In: ENTMME - Encontro Nacional de Tratamento de Minérios e Metalurgia Extrativa, 23, 2009, Gramado, RS. ENTMME: Volume I/Org. Irineu Antônio Schadach de Brum e Carlos Hoffmann Sampaio. Anais... São Paulo - SP: Associação Brasileira de Tratamento de Minérios e Metalurgia Extrativa, v. 1, p. 215-220, 2009.

5. DA SILVA, A. Q. N. 2015. 134 f. Modelagem da relação teor-recuperação da célula de flotação pneumática. Dissertação [Mestrado em Tecnologia Mineral]. Belo Horizonte, Pós-Graduação em Engenharia Metalúrgica, Materiais - Universidade Federal de Minas Gerais, 2015.

6. FEASBY, D. G. Investigation of Hallimond-tube flotation of low grade phosphate material. Process Report Minerals Research Laboratory, n. 1869; Book 212, 1966.

7. GUIMARÃES JÚNIOR, P.; SILVA, A. C.; SILVA, E. M. S. A influência da granulometria no arraste hidráulico em tubo de Hallimond. In: ENTMME - Encontro Nacional de Tratamento de Minérios e Metalurgia Extrativa, 26, 2015, Poços de Caldas, MG. ENTMME: Volume II/Org. Daniela Gomes Horta et al. Anais... São Paulo - SP: Associação Brasileira de Tratamento de Minérios e Metalurgia Extrativa, v. 2, p. 267-275, 2015.

8. GUVEN, O.; OZDEMIR O.; KARAAGACLIOGLU, I. E.; CELIK, M. S. Surface morphologies and floatability of sand-blasted quartz particles. Minerals Engineering, v. 70, p. 1-7, 2015.

9. MANGRAVITE JÚNIOR, F. J.; CASSEL, E. A.; MATIJEVIĆ, E. The Microflotation of Silica. Journal of Colloid and Interface Science, v. 39, n. 2, p. 357-366, 1972.

10. MARTINS, J.; SANTOS, A. R.; JUNIOR, G. G. O.; FREITAS, A. P.; PINTO, J. R.; SACRAMENTO, L. C. Efeito da granulometria do mineral na flotação convencional de quartzo puro. In: ENTMME Encontro Nacional de Tratamento de Minérios e Metalurgia Extrativa, 19, 2002, Recife, PE. ENTMME: Volume I/Org. Carlos Adolpho Magalhães Baltar et al. Anais... São Paulo - SP: Associação Brasileira de Tratamento de Minérios e Metalurgia Extrativa, v. 1, p. 291-294, 2002.

11. PINHEIRO, V. S.; BALTAR, C. A. M.; LEITE, J. Y. P. Influência da qualidade da água na flotação de quartzo com amina. Holos, n. 3, p. 28-36, 2010. 
12. REIS, J. L. M.; DA SILVA, F. L.; FREITAS JÚNIOR, L. R.; PEREIRA, C. A. Abordagem estatítica de Plackett-Burman na avaliação da microflotação de quartzo com dodecilamina. In: ENTMME Encontro Nacional de Tratamento de Minérios e Metalurgia Extrativa, 26, 2015, Poços de Caldas, MG. ENTMME: Volume II/Org. Daniela Gomes Horta et al. Anais... São Paulo - SP: Associação Brasileira de Tratamento de Minérios e Metalurgia Extrativa, v. 2, p. 305-313, 2015.

13. RODRIGUES, O. M. S.; ARAÚJO, A. C.; PERES, A. E. C. Microflotação de caulinita utilizando aminas. REM: Revista Escola de Minas, v. 63, n.4, p. 661-666, 2010.

14. RODRIGUES, T. S.; RODRIGUES, O.M.S.; NOVAIS, L. R.; PEREIRA, C. A.; PERES, A.E.C. Quantificação de arraste hidrodinâmico na microflotação. In: ENTMME - Encontro Nacional de Tratamento de Minérios e Metalurgia Extrativa, 24, 2013, Salvador, BA. ENTMME: Volume I/Org. Luiz Rogério Pinho de Andrade Lima. Anais... São Paulo - SP: Associação Brasileira de Tratamento de Minérios e Metalurgia Extrativa, v. 1, p. 355-359, 2011.

15. ROJAS, C. E. B. 2009. Reciclagem de sucata de jóias para recuperação hidrometalúrgica de ouro e prata. [Dissertação de Mestrado]. Pós-Graduação em Engenharia Metalúrgica e de Minas da Universidade Federal de Minas Gerais, Belo Horizonte; 2009.

16. SILVA, R. R. R. 2008. 153 f. Interação entre surfatantes na flotação de minérios de ferro. [Tese de Doutorado]. Pós-Graduação em Engenharia Metalúrgica e de Minas da Universidade Federal de Minas Gerais, Belo Horizonte; 2008.

17. TABOSA, E. O. 2007. 117 f. Flotação com reciclo de concentrados (frc) para recuperação de finos de minérios: fundamentos e aplicações. Dissertação [Mestrado em Tecnologia Mineral e Metalurgia Extrativa]. Porto Alegre, Pós-Graduação em Engenharia de Minas, Metalúrgica e de Materiais - Universidade Federal do Rio Grande do Sul, 2007.

18. ULUSOY, U.; YEKELER, M.; HIÇYILMAZ, C. Determination of the shape, morphological and wettability properties of quartz and their correlations. Minerals Engineering, v. 16, p. 951-964, 2003.

19. VICARIO, G. Metodo di Yates diretto ed inverso per piani fattoriali complete e misti. Statística Applicata, v. 8, n. 4, p. 741-755, 1996.

20. YATES, F. Complex experiments. Journal of the Royal Statistical Society, v. 2, n. 2, p. 181-247, 1935.

21. YATES, F. The design and analysis of factorial experiments. Technical Communication $\mathrm{n}=35$, Imperial Bureau of Soil Science, Harpenden, 1937. 